\title{
Curvature-constrained Directional-cost Paths in the Plane
}

\author{
Alan J. Chang · Marcus Brazil . \\ J. Hyam Rubinstein · Doreen A. Thomas
}

Received: date / Accepted: date

\begin{abstract}
This paper looks at the problem of finding the minimum cost curvature-constrained path between two directed points where the cost at every point along the path depends on the instantaneous direction. This generalises the results obtained by Dubins for curvatureconstrained paths of minimum length, commonly referred to as Dubins paths. We conclude that if the reciprocal of the directional-cost function is strictly polarly convex, then the forms of the optimal paths are of the same forms as Dubins paths. If we relax the strict polar convexity to weak polar convexity, then we show that there exists a Dubins path which is optimal. The results obtained can be applied to optimising the development of underground mine networks, where the paths need to satisfy a curvature constraint and the cost of development of the tunnel depends on the direction due to the geological characteristics of the ground.

Keywords Curvature constraint - Dubins paths · Path optimization - Directional cost · Anisotropic velocity · Pontryagin's Minimum Principle

Alan J. Chang

Department of Mechanical Engineering, The University of Melbourne, Victoria 3010, Australia

Tel.: +61-3-83446661

Fax: +61-3-83444290

E-mail: a.chang5@pgrad.unimelb.edu.au

Marcus Brazil

Department of Electrical and Electronic Engineering, The University of Melbourne, Victoria 3010, Australia Tel.: +61-3-83443829

Fax: +61-3-83446678

E-mail: brazil@unimelb.edu.au

J. Hyam Rubinstein

Department of Mathematics and Statistics, The University of Melbourne, Victoria 3010, Australia

Tel.: +61-3-83445548

Fax: +61-3-83444599

E-mail: rubin@ms.unimelb.edu.au

Doreen A. Thomas

Department of Mechanical Engineering, The University of Melbourne, Victoria 3010, Australia

Tel.: +61-3-83446663

Fax: +61-3-83444290

E-mail: doreen.thomas@unimelb.edu.au
\end{abstract}




\section{Introduction}

The problem studied in this paper is that of finding a minimum cost curvature-constrained path between two directed points where the cost at every point along the path depends on the instantaneous direction. The problem is properly defined later in section 2.3. In short, we wish to solve the problem of:

$$
\min _{E \in \mathbb{P}_{p q}} \int_{E} c(\alpha) \mathrm{d} s
$$

for some given directional-cost function $c(\alpha)$, where $\mathbb{P}_{p q}$ denotes the set of all curvatureconstrained paths between two directed points $p$ and $q$.

The motivation for studying this problem stems from the issue of directional faulting in the ground where an underground mine is to be developed. The resulting problem is also interesting in its own right. The costs associated with an underground mine tunnel are mostly made up of development, haulage, and other maintenance costs. The faulting results in regions of ground where the cost of development (both tunnelling and support) is significantly more in certain directions than others due to a required increase in time and resources to safely tunnel and provide extra support. Haulage and maintenance on the other hand is generally independent of the faulting, and simply depends on the length. The formulation we adopt in this paper is able to handle all of these costs, as the effect of haulage and maintenance would be just adding a constant to the directional-cost function of development.

Variations of the problem of finding the shortest curvature-constrained path between two directed points introduced by Dubins [5] have been extensively studied in the literature. A well-known extension is the problem of allowing the path to correspond to a vehicle which can move both forwards and backwards in [10]. More relevant extensions include [11] where the path crosses weighted regions of different velocity and [8] where the velocity of the airplane is affected by a constant wind. Also, [3] and [12] study the problem of classifying when a particular Dubins path is optimal for a given pair of directed points.

The major tools employed in tackling this problem are adapted from Dubins [5] and Boissonnat [1]. By transforming the minimum weighted length problem into a minimum time problem, it becomes clear that the reciprocal of the directional-cost function becomes the object of interest in characterising the forms of optimal paths that have to be considered. We denote this reciprocal as the velocity function, and introduce the concepts of strictness and polar convexity. A polarly convex (PC) velocity function means that the triangle inequality holds for cost of paths. This paper focuses on the forms of optimal paths that result when we consider PC velocity functions. A strict velocity function is one where we do not have an interval of directions where the triangle inequality is satisfied by equality. We distinguish between strictly polarly convex (SPC) where the strict triangle inequality holds, and weakly polarly convex (WPC) where the triangle inequality could be satisfied by equality.

The structure of this paper is as follows. The first step is to apply Pontryagin's Minimum Principle as in [1] to conclude that any optimal path must only consist of maximum curvature arcs and straight segments, given any strict velocity function. We then prove some basic properties of the cost of paths subjected to SPC velocity functions. Applying these properties allows us to generalise the results of [5] to conclude that the forms of optimal paths are the same as Dubins paths, if the velocity function is SPC. Finally, we show that there always exist an optimal path which is a Dubins path, if the velocity function is WPC.

Empirical studies of the relationship between rock strength and fault orientation relative to the tunnelling direction such as [6] and [7] demonstrate that there is a relationship between the required support cost and the direction in which the tunnel is developed. As the 
problem of finding shortest underground mine networks with this additional consideration is not yet solved, common practice is to use experience and intuition to account for this. Understanding exactly how to solve this problem mathematically will enable the geological information to be incorporated into existing algorithms such as in [2]. By incorporating directional dependency of cost into the algorithms, the resulting output will be much more accurate and helpful for the preliminary design of underground mine networks.

During the course of writing this paper, it was brought to our attention that similar work was being done concurrently by Dolinskaya in [4]. The application in their case was for naval path planning but is mathematically formulated in similar manner. In their work, they consider an anisotropic minimum radius of curvature function, as well as an anisotropic velocity function. The motivation and future direction in which their work is being developed is different from our work.

\section{Background}

We first define what we mean by curvature-constrained paths.

\subsection{Curvature-constrained paths}

In the following, $a, b \in \mathbb{R}^{2}$ and $p, q \in \mathbb{R}^{2} \times \mathbb{R} / 2 \pi$. Also, $C^{1}$ (resp. $C^{2}$ ) will mean that the first (resp. second) derivative exists and is continuous.

A path from $a$ to $b$ is a directed piecewise $C^{1}$ curve from $a$ to $b$. We parametrise our paths by arc-length, $E:\left[0, t_{f}\right] \rightarrow \mathbb{R}^{2}$ with $E(0)=a$ and $E\left(t_{f}\right)=b$. The direction $\alpha \in \mathbb{R} / 2 \pi$ at a differentiable point of a path $E(t)$ is given by the polar angle of the tangent at the point in the direction of increasing $t$. At the endpoints, the directions are taken as the respective limits of $\alpha$.

Given a minimum turning circle radius $R>0$, a curvature-constrained path between two directed points $p, q$ is a $C^{1}$ path that is piecewise $C^{2}$, where the absolute curvature everywhere along the path is bounded by $1 / R$, and the given directions at the start and end points coincide with the directions of $p$ and $q$ respectively. Without loss of generality, we assume the minimum turning circle radius $R=1$ in this entire paper. Let $\mathcal{C}$ be a label denoting an arc of maximum curvature of length less than $2 \pi$. Let $\mathcal{S}$ be a label denoting a straight line segment.

A CS-path is a curvature-constrained path $E:\left[0, t_{f}\right] \rightarrow \mathbb{R}^{2}$ such that there exist $t_{0}, \cdots, t_{n}$ such that $t_{0}=0$ and $t_{n}=t_{f}, t_{i-1}<t_{i}$ for $i=1, \cdots, n$, where $E$ is not twice differentiable at $t_{i}$ for $i=1, \cdots, n$, and each subpath $E_{i}:\left[t_{i-1}, t_{i}\right] \rightarrow \mathbb{R}^{2}$ is either a $\mathcal{C}$ arc or $\mathcal{S}$ segment. The form of such a CS-path is then the sequence of $\mathcal{C}$ and $\mathcal{S}$ labels in ascending order of $i$. The sense of a $\mathcal{C}$ can be further specified using the labels $\mathcal{L}$ and $\mathcal{R}$ for left-turning and right-turning arcs respectively. In the form of a CS-path, any consecutive $\mathcal{C}$ arcs must be of opposite sense due to the condition that the CS-path is differentiable but not twice differentiable at the point in between two consecutive labels. This condition also implies that there will never be consecutive $\mathcal{S}$ labels in the form of a CS-path.

Dubins [5] first studied the problem of finding the shortest curvature-constrained path between any two given directed points in $\mathbb{R}^{2}$ and proved the following theorem.

Theorem 1 Given any two directed points $p, q \in \mathbb{R}^{2} \times \mathbb{R} / 2 \pi$ and a prescribed minimum radius of curvature $R$, the shortest curvature-constrained path is a CS-path with one of the following forms. 
1. $\mathcal{C S C}$

2. $\mathcal{C} \overline{\mathcal{C}}$

3. any degeneracies of the two forms

where $\overline{\mathcal{C}}$ denotes a $\mathcal{C}$ arc of length greater than $\pi R$

CS-paths of the forms described in Theorem 1 will be referred to as Dubins paths. Note that there are at most 6 distinct Dubins paths for any pair $p, q$ because of the different sense of the $\mathcal{C}$ arcs, but they do not all necessarily exist and degeneracies may occur.

Theorem 1 was later proved again by Boissonnat et al. [1] by formulating it as a control problem and applying Pontryagin's Minimum Principle. A more detailed version of [1] can be found in [13]. We summarise the key ideas here as we will need to use the control formulation for one of the main results.

\subsection{Control Formulation}

We summarise the necessary background for applying Pontryagin's Minimum Principle from [9]. Note that we are presenting a simplified version of the principle as that is sufficient for this paper. We also present the minimum principle as opposed to the maximum principle originally formulated in [9], since it is commonly stated in the minimum principle form in the current literature.

Consider a system of differential equations with prescribed boundary conditions as in (1), where $x(t)$ denotes the states of the system, and the piecewise continuous function $u:[0, T] \rightarrow U$ is the control we want to choose, for some convex and compact $U$. The functions $f_{j}$ are continuous in the variables $x, u$ and continuously differentiable with respect to $x$.

$$
\begin{aligned}
& \dot{x_{j}}=f_{j}(x(t), u(t)), x(0)=x^{i}, x(T)=x^{f} \text { for } j=1, \cdots, n \\
& \text { where } x(t)=\left(x_{1}(t), \cdots, x_{n}(t)\right) \text { and } \dot{z} \text { denotes } \mathrm{d} z / \mathrm{d} t
\end{aligned}
$$

We restrict ourselves to only considering the controls that satisfy the boundary conditions. Such controls will be referred to as admissible controls. Since we are interested in time optimality, the relevant cost function $J(u)$ is of the form shown in (2) below. We aim to find an admissible control which minimises $J$, the total time to start from $x^{i}$ and end at $x^{f}$.

$$
J(u)=\int_{0}^{T} \mathrm{~d} t
$$

To state the minimum principle, we need to introduce another system of equations in the auxiliary variables $\psi_{1}, \cdots, \psi_{n}$ as follows.

$$
\dot{\psi}_{j}=-\sum_{k=1}^{n} \frac{\partial f_{j}}{\partial x_{k}} \psi_{k} \text { for } j=1, \cdots, n
$$

Let the Hamiltonian $H$ be defined as follows.

$$
H(\psi, x, u)=\sum_{k=1}^{n} \psi_{k} f_{k}(x, u)
$$

For fixed $\psi, x, H$ is a function of $u$. Let $M(\psi, x)$ denote the lower bound of the values of $H$. 


$$
M(\psi, x)=\inf _{u \in U} H(\psi, x, u)
$$

We can then state the minimum principle as follows.

Theorem 2 If $u(t)$ is an optimal admissible control, there exists a nonzero continuous vector function $\psi(t)$ satisfying (3), such that $\forall t \in[0, T]$,

1. $H(\psi(t), x(t), u(t))=M(\psi(t), x(t))=$ constant

2. $M(\psi(t), x(t)) \leq 0$

\subsection{Directional Cost}

We now introduce directional-cost to curvature-constrained paths. Some basic terms which will be used consistently throughout this paper are defined in this section. Let $\mathbb{R}_{+}$denote the set of all strictly positive real numbers $\{x \in \mathbb{R}: x>0\}$.

A directional-cost function is a continuous, piecewise $C^{2}$ function $c: \mathbb{R} / 2 \pi \rightarrow \mathbb{R}_{+}$. The corresponding velocity function $v: \mathbb{R} / 2 \pi \rightarrow \mathbb{R}_{+}$is given by $v(\alpha)=1 / c(\alpha)$, which is also continuous and piecewise $C^{2}$. The interpretation of the velocity function in the context of directional-cost is a measure of the distance that can be travelled in the direction $\alpha$ at the cost of one unit.

Given a path $E$ and a directional-cost function $c$,

- the length of $E, L(E)$, is given by $L(E)=\int_{E} \mathrm{~d} s$.

$-E$ is degenerate if it has zero length.

- the cost of $E, T(E)$, is given by $T(E)=\int_{E} c(\alpha) \mathrm{d} s$

Let $\mathbb{P}_{p q}$ denote the set of all curvature-constrained paths between two directed points $p$ and $q$. An optimal path from $p$ to $q$ is a path $E \in\left\{P \in \mathbb{P}_{p q}: T(P) \leq T(Q) \forall Q \in \mathbb{P}_{p q}\right\}$.

Our problem involves identifying an optimal path between given start and end directed points, subject to a given velocity function. It can be seen that by the way the problem has been posed, the problem of finding the minimum total cost path is equivalent to a problem of finding the minimum total time for a vehicle to travel from $p$ to $q$ if the velocity of the vehicle depends on the direction it is facing at any point in time. This is the motivation for calling the reciprocal of the directional-cost function a velocity function.

\section{Application of Pontryagin's Minimum Principle}

In [1], the problem is formulated as a minimum time problem by having a cost function that is simply the integral of 1 over the path. We could simply modify the cost function to be the integral of $c(\alpha)$ instead, and indeed this will yield the same result. However, we choose to formulate the problem differently, keeping the cost function the same, while incorporating the directional-cost by making the velocity of the vehicle $v(\alpha)$. Both these approaches are mathematically identical, but the latter provides additional insight which is helpful for motivating the later sections. We first consider velocity functions that are strict as defined below.

Given a velocity function $v$, let $K=v v^{\prime \prime}-2 v^{\prime 2}-v^{2}$ which is defined for almost all $\alpha$ since $v$ is piecewise $C^{2}$. Since $K(\alpha)=\kappa(\alpha)\left(v^{2}+v^{\prime 2}\right)^{\frac{3}{2}}$ where $\kappa(\alpha)$ is the signed curvature of the polar function $v$, the sign of $K$ gives the sign of the curvature of $v$. In particular, if 
$K=0$ on an interval $\left[\alpha_{1}, \alpha_{2}\right]$, this corresponds to $v$ being a straight line in polar coordinates from $\left(v\left(\alpha_{1}\right), \alpha_{1}\right)$ to $\left(v\left(\alpha_{2}\right), \alpha_{2}\right)$. We discuss the implications of this in Section 4.

A velocity function is strict if there are no (non-trivial) intervals $\left[\alpha_{1}, \alpha_{2}\right]$ where $K(\alpha)=0$ $\forall \alpha \in\left[\alpha_{1}, \alpha_{2}\right]$.

Lemma 1 For any given pair of directed points $p, q \in \mathbb{R}^{2} \times[0,2 \pi)$ and strict velocity function $v$, any optimal path is necessarily a CS-path.

Proof First, let us consider the case where $v$ is strict and $C^{2}$ everywhere. Let the states $x(t), y(t)$ and $\alpha(t)$ represent the coordinates and direction of the path at time $t$. Let $u(t)$ be the control variable that governs the instantaneous curvature at time $t$. Recall that without loss of generality, we let the minimum radius of curvature be 1 and hence, $u \in[-1,1]$. Let $p=\left(x^{i}, y^{i}, \alpha^{i}\right)$ and $q=\left(x^{f}, y^{f}, \alpha^{f}\right)$. Our problem can then be formulated in the following manner.

$$
\begin{gathered}
\dot{x}=v(\alpha) \cos (\alpha), x(0)=x^{i}, x(T)=x^{f} \\
\dot{y}=v(\alpha) \sin (\alpha), \quad y(0)=y^{i}, y(T)=y^{f} \\
\dot{\alpha}=v(\alpha) u \quad, \alpha(0)=\alpha^{i}, \alpha(T)=\alpha^{f} \\
J(u)=\int_{0}^{T} \mathrm{~d} t
\end{gathered}
$$

Let $\psi_{x}, \psi_{y}$ and $\psi_{\alpha}$ be the corresponding auxiliary variables. From (3) we get the following.

$$
\begin{aligned}
\dot{\psi}_{x} & =0 \\
\dot{\psi}_{y} & =0 \\
\dot{\psi}_{\alpha} & =\psi_{x} v(\alpha) \sin \alpha-\psi_{x} v^{\prime}(\alpha) \cos \alpha-\psi_{y} v(\alpha) \cos \alpha-\psi_{y} v^{\prime}(\alpha) \sin \alpha-v^{\prime}(\alpha) \psi_{\alpha} u
\end{aligned}
$$

From (4) we get an expression for the Hamiltonian $H$ as follows.

$$
H=\psi_{x} v(\alpha) \cos (\alpha)+\psi_{y} v(\alpha) \sin (\alpha)+v(\alpha) \psi_{\alpha} u
$$

Define $\lambda$ and $\phi$ by $\lambda=\sqrt{\psi_{x}^{2}+\psi_{y}^{2}} \geq 0, \tan \phi=\psi_{y} / \psi_{x}, \phi \in[0,2 \pi)$ so that $\psi_{x}=\lambda \cos \phi$, $\psi_{y}=\lambda \sin \phi$. Observe that $\psi_{x}$ and $\psi_{y}$ are constant on $[0, T]$, so it follows that $\lambda$ and $\phi$ are also constant on $[0, T]$. We can then rewrite $H$ and $\dot{\psi}_{\alpha}$ as follows.

$$
\begin{aligned}
H & =\lambda v(\alpha) \cos (\alpha-\phi)+v(\alpha) \psi_{\alpha} u \\
\dot{\psi}_{\alpha} & =\lambda v(\alpha) \sin (\alpha-\phi)-\lambda v^{\prime}(\alpha) \cos (\alpha-\phi)-v^{\prime}(\alpha) \psi_{\alpha} u
\end{aligned}
$$

By the minimum principle (Theorem 2), in order for the control $u$ to be optimal, either:

1. $\partial H / \partial u \neq 0 \Rightarrow u= \pm 1$ so the path is an arc of radius 1 ; or

2. $\partial H / \partial u=v(\alpha) \psi_{\alpha}=0 \Rightarrow \psi_{\alpha}=0$ 
We now show that $u=0$ is for the second case. Since $\psi_{\alpha}=0$, we have $\dot{\psi}_{\alpha}=0$ as well. Note that $\lambda \neq 0$ since $\lambda=0 \Rightarrow\left(\psi_{x}, \psi_{y}, \psi_{\alpha}\right)=(0,0,0)$, which violates the condition that this vector must be non-zero. Rearranging (10) then gives the following.

$$
\alpha=\left\{\begin{array}{l}
\phi+\arctan \left(v^{\prime}(\alpha) / v(\alpha)\right) \text { or } \\
\phi+\arctan \left(v^{\prime}(\alpha) / v(\alpha)\right)+\pi
\end{array}\right.
$$

Differentiating either of the two possibilities for $\alpha$ with respect to $t$ and recalling $\dot{\alpha}=$ $v(\alpha) u$ gives the following.

$$
u\left(v v^{\prime \prime}-2 v^{\prime 2}-v^{2}\right)=0
$$

Recall that $K=v v^{\prime \prime}-2 v^{\prime 2}-v^{2}$ where the sign of $K$ gives the sign of the curvature of $v$. When $K \neq 0$, it follows that $u=0$. Where we are unable to guarantee $u=0$ is at some time $t^{*}$ when $K\left(\alpha\left(t^{*}\right)\right)=0$. Since $v$ is strict, for any $\alpha^{*}$ where $K\left(\alpha^{*}\right)=0$, there exists $\varepsilon>0$ such that $K(\alpha) \neq 0 \forall \alpha \in\left(\alpha^{*}-\varepsilon, \alpha^{*}+\varepsilon\right) \backslash\left\{\alpha^{*}\right\}$. If $u\left(t^{*}\right) \neq 0, \alpha$ is changing when $t=t^{*}$ since $\dot{\alpha}=u$. Hence, there exists $\delta>0$ such that for any $t \in\left(t^{*}, t^{*}+\delta\right), K(\alpha(t)) \neq 0$. Hence, $u$ is never non-zero for time intervals of non-zero length. Alternatively, $u\left(t^{*}\right)=0$ would correspond to an $\mathcal{S}$ subpath in the direction $\alpha\left(t^{*}\right)$. Hence, any optimal path is a CS-path if $v$ is $C^{2}$ everywhere and there are only a finite number of points where $K=0$.

Suppose that $v$ is piecewise $C^{2}$ instead of $C^{2}$ everywhere. It follows that there are only a finite number of directions $\alpha_{i}$ where $v\left(\alpha_{i}\right)$ is not twice differentiable. By applying a similar argument as above for the directions where $K=0$, we conclude that any optimal path is a CS-path if $v$ is a strict velocity function.

\section{Polarly Convex Velocity Function}

In the previous section, we established that any optimal path is a CS-path, given a strict velocity function $v$. We are interested in establishing the forms of optimal paths for a given $v$. In [5], Dubins paths are established to be optimal through Euclidean geometric arguments. Here, these arguments are no longer available to us due to the nature of the velocity function. However, if we assume that the velocity function $v$ is strictly polarly convex, by which we mean that $v$ is strict and it maps out the boundary of a convex region in $\mathbb{R}^{2}$ (in polar coordinates), then it can be shown that the strict triangle inequality holds, which is sufficient to re-establish the main results of Dubins.

In this section, we show that strict polar convexity of the velocity function implies that the forms of optimal curvature-constrained paths are the same as Dubins paths. However, an example is given to show that a shortest curvature-constrained path is not necessarily an optimal path for a particular choice of start and end directed points, even if the velocity function is strictly polarly convex. Furthermore, we show that by relaxing the strict convexity to weak convexity, there still always exists a Dubins path which is an optimal path.

The practical implication of these results is that the optimal path can be constructed by computing the cost of the (up to 6) Dubins paths between any two directed points, and selecting the path of lowest cost among the Dubins paths. For the original Dubins problem of constructing the shortest path, there have been works done to exactly determine which of the Dubins paths are optimal based on the relative positions and orientations of directed points, such as in [3] and [12]. Based on their results, the partitioning of the configuration space is non-trivial, and hence, if we consider the extension to their problem of introducing a polarly convex velocity function, it would be infeasible to obtain an elegant partitioning to the configuration space in general. 


\subsection{Polar convexity}

A straight path $a b$ is a path from $a$ to $b$ where $\alpha$ is constant along the entire path and equal to $\alpha_{a b}$. A polygonal path is a path $a_{1} \cdots a_{k+1}$ made up of $k$ straight paths $a_{1} a_{2}, a_{2} a_{3}, \cdots, a_{k} a_{k+1}$.

Given a velocity function $v$, the velocity set $V$ is the subset of $\mathbb{R}^{2}$ enclosed by the polar plot of $v$ represented in polar coordinates by Given a velocity function $v$, the velocity set $V$ is the subset of $\mathbb{R}^{2}$ enclosed by the polar plot of $v$ represented in polar coordinates by

$$
V=\{e=(\varepsilon(\alpha), \alpha): \varepsilon(\alpha) \in[0, v(\alpha)] \forall \alpha \in[0,2 \pi)\}
$$

The velocity set can be interpreted in the context of directional-cost as the set of all points that can be reached by a single straight path from the origin at the cost of no greater than one unit.

Let $\operatorname{conv}(S)$ denote the convex hull of the set $S$. A velocity function $v$ is

- polarly convex $(P C)$ if $V=\operatorname{conv}(V)$.

- strictly polarly convex (SPC) if it is $\mathrm{PC}$ and strict.

- weakly polarly convex (WPC) if it is $\mathrm{PC}$ but not strict.

- polarly non-convex $(P N C)$ if it is not PC.

Let $\mathbb{E}^{2}=\left(\mathbb{R}^{2},\|\cdot\|_{e}\right)$ where $\|\cdot\|_{e}$ is the Euclidean norm. If $v$ is symmetric i.e. $v(\alpha)=$ $v(\alpha+\pi)$, the cost of a straight path $a b$ in $\mathbb{E}^{2}$ subject to a PC function $v$ is the same as the norm of $a b$ in a normed space $\mathbb{R}^{2}$ equipped with a norm $\|\cdot\|_{v}$ that has a unit ball $V$ since $T(a b)=L(a b) / v\left(\alpha_{a b}\right)=\|b-a\|_{v}$. By relaxing the symmetry property of the normed space but retaining the triangle inequality property, it can be seen that the cost of straight paths and polygonal paths subject to any PC velocity function $v$ satisfies the triangle inequality

$$
T(a e b) \geq T(a b)
$$

Similarly, the cost of straight paths and polygonal paths subject to an SPC velocity function satisfies the strict triangle inequality that is

$$
T(a e b)>T(a b) \text { if } e \text { does not lie on } a b
$$

Let $f(z-0)=\lim _{x \rightarrow z^{-}} f(x)$ and $f(z+0)=\lim _{x \rightarrow z^{+}} f(x)$. Recall that $K=v v^{\prime \prime}-2 v^{\prime 2}-v^{2}$.

Property 1 It can be shown using basic properties of curvature and convexity of functions that $v(\alpha)$ is $\mathrm{PC}$ iff

1. $K(\alpha) \leq 0$ where $v(\alpha)$ is twice differentiable; and

2. $v^{\prime}\left(\alpha_{i}-0\right) \geq v^{\prime}\left(\alpha_{i}+0\right)$ at the points $\alpha_{i}$ where $v(\alpha)$ is not twice differentiable

Equivalently, in terms of $c=1 / v$, we have that $v(\alpha)$ is PC iff

1. $c^{\prime \prime}(\alpha)+c(\alpha) \geq 0$ where $c(\alpha)$ is twice differentiable; and

2. $c^{\prime}\left(\alpha_{i}-0\right) \leq c^{\prime}\left(\alpha_{i}+0\right)$ at the points $\alpha_{i}$ where $c(\alpha)$ is not twice differentiable

We now prove some basic properties of paths subject to an SPC velocity function. Let $\operatorname{bd}(S)$ denote the boundary of $S$. In the following, we refer to points in $\mathbb{R}^{2}$ as being represented in polar coordinates. A direction $\alpha^{\prime}$ is a convex direction if $\left(v\left(\alpha^{\prime}\right), \alpha^{\prime}\right) \in \operatorname{bd}(\operatorname{conv}(V))$. A direction $\alpha^{*}$ is a concave direction if $\left(v\left(\alpha^{\prime}\right), \alpha^{\prime}\right) \notin \mathrm{bd}(\operatorname{conv}(V))$.

Lemma $2 v$ is SPC iff the cost of the straight path between any two points is less than the cost of any other path between the two points. 
Proof $(\Leftarrow)$ The contrapositive is that there exists a path between two points that is of lower or equal cost than the straight path between the two points if $v(\alpha)$ is PNC or WPC. First, suppose that it is PNC. Let $a$ be the origin, and $b$ be an arbitrary distance in a concave direction from $a$. Choose $e$ so that $\alpha_{a e}$ and $\alpha_{e b}$ are the first convex directions encountered when searched for in the anticlockwise and clockwise directions from $\alpha_{a b}$ (clearly such a $e$ exists). Let $g(\alpha)$ be a PC velocity function such that its velocity set $G=\operatorname{conv}(V)$. Since $\alpha_{a b}$ is a concave direction, $g\left(\alpha_{a e}\right)=v\left(\alpha_{a e}\right)$ and $g\left(\alpha_{e b}\right)=v\left(\alpha_{e b}\right)$ while $g\left(\alpha_{a b}\right)>v\left(\alpha_{a b}\right)$. Let $T_{v}$ and $T_{g}$ denote the costs of paths subjected to velocity functions $v$ and $g$ respectively. Note that $e$ was chosen so that $T_{g}(a e b)=T_{g}(a b)$, so $T_{v}(a e b)=T_{g}(a e b)=T_{g}(a b)<T_{v}(a b)$. On the other hand if $v(\alpha)$ is WPC, it follows from its definition that there exists $a, b, e$ where $e$ does not lie on $a b$, such that $T(a e b)=T(a b)$.

$(\Rightarrow)$ Since $v$ is SPC, the strict triangle inequality (13) holds. Let $E(t)$ be a path with $E(0)=a$ and $E\left(t_{f}\right)=b$ that is not $a b$. Let $e=E\left(t_{f} / 2\right)$ so if $e$ lies on $a b$ then $T(a e b)=T(a b)$, otherwise $T(a e b)>T(a b)$. Hence in general, $T(a e b) \geq T(a b)$. This bisection argument can be repeated infinitely on the new straight paths generated to show that $T(E) \geq T(a b)$. The first few steps of this procedure are illustrated diagrammatically in Figure 1. The convergence of this polygonal path to $E(t)$ is guaranteed by the properties of Euclidean space. Observe that since $T(a b)=T(E)$ can only occur if the inequality is an equality at every step, that would imply that $E=a b$ which is a contradiction and hence, $T(a b)<T(E)$.
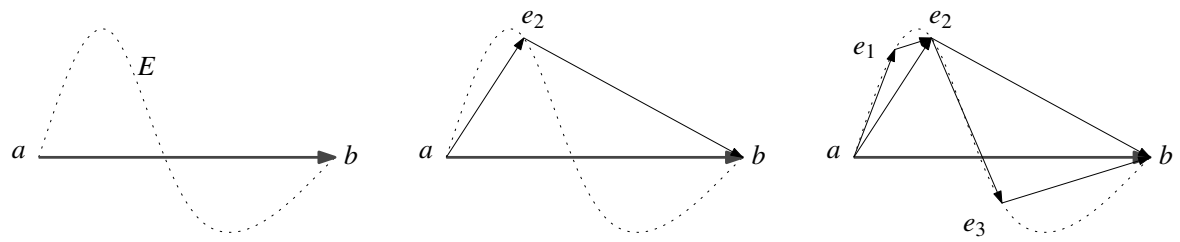

Fig. $1 T(a b) \leq T\left(a e_{2} b\right) \leq T\left(a e_{1} e_{2} e_{3} b\right) \leq \ldots \leq T(E)$

A convex path is a piecewise $C^{2}$ path that lies on the boundary of its own convex hull. A supporting line to a convex path at a point $e$ along the convex path, is any supporting line of the convex hull of the path at $e$. Given a convex path represented by $E:\left[0, t_{f}\right] \rightarrow \mathbb{R}^{2}$, an outer normal $n_{e}$ of a point $e=E\left(t_{e}\right)$ for some $t_{e} \in(0,1)$, is the normal unit vector to a supporting line at $e$ that points away from the convex hull. The outer normal also divides the supporting line at $e$ into two halves, referred to as being the supporting line on a particular side of the outer normal. Note that the outer normal is not unique if $e$ is not a differentiable point. For the following arguments, this will not be an issue, as if there are multiple choices of $n_{e}$, picking any one will work.

A path lies on the outer side of a convex path if the path is formed by continuously deforming the convex path, without entering the convex hull of the convex path,and is not the convex path itself. It follows from these definitions that a supporting line at any point along a convex path $E$ never intersects the interior of the convex hull of $E$ and must always intersect a path that lies on the outer side of $E$ on both sides of the outer normal at that point.

Lemma $3 v$ is SPC iff the cost of any path lying on the outer side of a convex path is greater than the cost of the convex path.

Proof $(\Leftarrow)$ The contrapositive is that there exists a path lying on the outer side of a convex path that is of lower or equal cost than the convex path if $v$ is PNC or WPC. This follows 
immediately by applying the proof of Lemma 2 in the $(\Leftarrow)$ direction by taking the straight path to be the convex path.

$(\Rightarrow)$ Let $E(t)$ be a convex path with $E(0)=a$ and $E\left(t_{f}\right)=b$. Let $e=E\left(t_{f} / 2\right)$ and $G(e)$ be a supporting line at $e$. Let $E_{1}(t)$ be a path lying on the outer side of $E(t)$ with $E_{1}(0)=a$ and $E_{1}\left(t_{f}\right)=b$.

By definition, $G(e)$ does not ever intersect the interior of the convex hull of $E$, but has to intersect $E_{1}$ at least once on each side of $n_{e}$. Pick an intersection point from each side of the supporting line which is closest to $e, E_{1}\left(t_{1}\right)$ and $E_{1}\left(t_{2}\right)$, with $t_{2} \geq t_{1}$ (Note that in the degenerate case of $E^{\prime}$ coinciding locally with $E$ at $e$, these two points are the same).

Let $\hat{E}$ denote the subpath of $E_{1}$ from $E_{1}\left(t_{1}\right)$ to $E_{1}\left(t_{2}\right)$. By Lemma 2, $T\left(E_{1}\left(t_{1}\right) E_{1}\left(t_{2}\right)\right)<$ $T(\hat{E})$ if $\hat{E} \neq E_{1}\left(t_{1}\right) E_{1}\left(t_{2}\right)$. Hence, the cost of the new path $E_{2}$ formed by the subpath of $E_{1}$ from $a$ to $E_{1}\left(t_{1}\right)$, followed by $E_{1}\left(t_{1}\right) E_{1}\left(t_{2}\right)$, and then the subpath of $E_{1}$ from $E_{1}\left(t_{2}\right)$ to $q$, is less than or equal to the cost of $E_{1}$.

This bisection argument can now be repeated on the subpaths of $E_{2}$ lying on the outer side of the subpaths of $E$ from $a$ to $e$ and $e$ to $b$. By infinitely iterating this construction, the path converges to a path $E^{*}$ which is either the same as $E$ or contains $E$ as a subpath, and is of lesser or equal cost to $E_{1}$. Hence, $T\left(E_{1}\right) \geq T(E)$. The first few steps of this procedure are illustrated diagrammatically in Figure 2. The convergence is guaranteed by properties of Euclidean space. Observe that since $T\left(E_{1}\right)=T(E)$ can only occur if the inequality was satisfied by equality at every step, that would imply that $E_{1}=E$ which is a contradiction and hence, $T\left(E_{1}\right)>T(E)$.

In terms of lengths in $\mathbb{E}^{2}$ or equivalently, if $v(\alpha)=1$, this is a trivial observation, as it can be thought of physically as a string lying above a convex curve being deformed without penetrating the curve, which obviously only ever lengthens the string. This lemma generalises the concept to any arbitrary $\operatorname{SPC} v(\alpha)$.
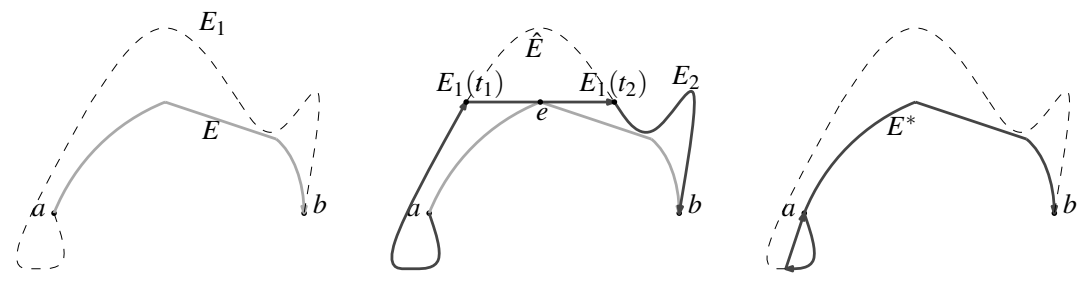

Fig. $2 T\left(E_{1}\right) \geq T\left(E_{2}\right) \geq \ldots \geq T\left(E^{*}\right) \geq T(E)$

\subsection{Optimal forms}

Lemma 3 gives us the fundamental tool for working with SPC velocity functions. We are now able to identify the forms of optimal paths. Recall that the form of a CS-path is given by a string of $\mathcal{C}$ and $\mathcal{S}$ labels representing arcs of radius 1 and straights respectively. We let $\overline{\mathcal{C}}$ denote a $\mathcal{C}$ arc of length greater than $\pi$ and $\underline{\mathcal{C}}$ denote a $\mathcal{C}$ arc of length no greater than $\pi$.

By adopting the methodology of Dubins [5] of showing that each of the following types of paths can be continuously deformed to a different path of lower cost, we will show that the optimal forms are Dubins paths.

1. $\mathcal{S C S}$ 
2. $\mathcal{C C S}$

3. $\mathcal{C C C}$

4. $\mathcal{C C C C}$

Lemma 4, Corollary 1 and Corollary 2 below are extensions of Dubins' proofs to a SPC $v(\alpha)$ instead of $v(\alpha)=1$. Note that since these arguments use the fact that a subpath of an optimal path must itself be optimal, we are able to arbitrarily shrink the lengths of the first and last arc or line segment to simplify the proofs.

Given a CS-path, we denote by $\mathbf{C}_{k}$ a particular subpath of the form $\mathcal{C}$ where $k \in \mathbb{Z}_{+}$is simply an index to distinguish different subpaths. $\underline{\mathbf{C}}_{k}, \overline{\mathbf{C}}_{k}, \mathbf{L}_{k}, \underline{\mathbf{L}}_{k}, \overline{\mathbf{L}}_{k}, \mathbf{R}_{k}, \underline{\mathbf{R}}_{k}, \overline{\mathbf{R}}_{k}, \mathbf{S}_{k}$ are defined similarly. A CS-path can be written as a concatenation of its subpaths traversed in order. A direction plot of a CS-path is a plot in polar coordinates of all the directions traversed during that path, plotted at increasing radii for different $\mathcal{C}$ arcs in the order traversed, with radial line segments representing $\mathcal{S}$ segments. This contains all the information required to compute the total cost of that path, provided the lengths of all $\mathcal{S}$ segments are separately specified. We introduce direction plots to make the following proofs' ideas clearer.

Given directed points $p=\left(x_{p}, y_{p}, \alpha_{p}\right), q=\left(x_{q}, y_{q}, \alpha_{q}\right)$, we let $p q$ denote a straight path from $\left(x_{p}, y_{p}\right)$ to $\left(x_{q}, y_{q}\right)$, and likewise for polygonal paths.

Lemma 4 If $v$ is $S P C$, then any $\mathcal{S C S}$ path is not optimal.

Proof To prove the result, it needs to be shown that a path of the form $\mathcal{S C S}$ can be improved. There are only two possible cases to consider, depending on the length of the $\mathcal{C}$ arc.

1. $\mathcal{S} \underline{\mathcal{S}}$

Without loss of generality, consider a path $\mathbf{S}_{1} \underline{\mathbf{R}}_{2} \mathbf{S}_{3}$ between directed points $p$ and $q$ as shown in Figure 3. Let the $\mathcal{R S R}$ path from $p$ to $q$ be $\mathbf{R}_{4} \mathbf{S}_{5} \mathbf{R}_{6}$. It follows by Lemma 3 that $T\left(\mathbf{S}_{1} \underline{\mathbf{R}}_{2} \mathbf{S}_{3}\right)>T\left(\mathbf{R}_{4} \mathbf{S}_{5} \mathbf{R}_{6}\right)$ since $\mathbf{R}_{4} \mathbf{S}_{5} \mathbf{R}_{6}$ is a convex path with $\mathbf{S}_{1} \underline{\mathbf{R}}_{2} \mathbf{S}_{3}$ lying on its outer side.

2. $\mathcal{S} \overline{\mathcal{C}} \mathcal{S}$

Without loss of generality, consider a path $\mathbf{S}_{1} \overline{\mathbf{R}}_{2} \mathbf{S}_{3}$ between directed points $p$ and $q$ with sufficiently short $\mathcal{S}$ segments of appropriate lengths so that $\mathbf{R}_{4} \mathbf{L}_{6}$, the $\mathcal{R} \mathcal{L}$ path from $p$ to $q$, exists as shown in Figure 4. Let $\mathbf{S}_{1}$ be $p \hat{p}$ and $\mathbf{S}_{3}$ be $\hat{q} q$. Since, $L\left(\mathbf{R}_{4}\right)>L\left(\overline{\mathbf{R}}_{2}\right)$, the subpath $\mathbf{R}_{5} \mathbf{L}_{6}$ from $r$ to $q$, where $L\left(\mathbf{R}_{5}\right)=L\left(\mathbf{R}_{4}\right)-L\left(\overline{\mathbf{R}}_{2}\right)$, exists. Since translation of a path does not affect its cost, the result is proven if $T\left(\mathbf{R}_{5}\right)+T\left(\mathbf{L}_{6}\right)<T\left(\mathbf{S}_{1}\right)+T\left(\mathbf{S}_{3}\right)-$ see Figure 5. Note that since the direction at $r$ and $q$ along $\mathbf{R}_{5} \mathbf{L}_{6}$ are both $\alpha_{\hat{q} q}$, it follows that $L\left(\mathbf{R}_{5}\right)=L\left(\mathbf{L}_{6}\right)$. It then follows by symmetry that $r q$ intersects $\mathbf{R}_{5} \mathbf{L}_{6}$ at $s$, where $\mathbf{R}_{5}$ ends at $s$ and $\mathbf{L}_{6}$ starts at $s$. Let the direction of $\mathbf{R}_{5} \mathbf{L}_{6}$ at $s$ be $\alpha_{s}$. From the direction plot of $\mathbf{R}_{5} \mathbf{L}_{6}$ shown in Figure 6, it is clear that $T\left(\mathbf{R}_{5}\right)=T\left(\mathbf{L}_{6}\right)$ since $\mathbf{R}_{5}$ and $\mathbf{L}_{6}$ both traverse the same set of directions. Let $t$ be the midpoint of $\hat{q} q$. By translating $\mathbf{S}_{1}$ so that it now begins at $r$, a polygonal path $r \hat{q} q$ is formed where $T(r \hat{q} q)=2 T(s t q)$ by similar triangles. By applying Lemma 3 to the convex $\operatorname{arc} \mathbf{L}_{6}$, it follows that $T\left(\mathbf{L}_{6}\right)<T(s t q)$. The result then follows since $T\left(\mathbf{R}_{5}\right)+T\left(\mathbf{L}_{6}\right)=2 T\left(\mathbf{L}_{6}\right)<2 T(s t q)=T(r \hat{q} q)=T\left(\mathbf{S}_{1}\right)+T\left(\mathbf{S}_{3}\right)$.

\section{Corollary 1 If $v$ is $S P C$, then any $\mathcal{C C S}$ path is not optimal.}

Proof This result follows from applying similar arguments to those in Lemma 4. If the path is of the form $\mathcal{C} \mathcal{C} S$, the argument for the $\mathcal{S} \mathcal{C} \mathcal{S}$ case can be applied by taking a sufficiently small subpath of the $\mathcal{C} \mathcal{C} \mathcal{S}$ path. Otherwise, consider a path $\mathbf{L}_{1} \overline{\mathbf{R}}_{2} \mathbf{S}_{3}$ and apply the argument for the $\mathcal{S} \overline{\mathcal{C}} \mathcal{S}$ case, replacing $\mathbf{S}_{1}$ with $\mathbf{L}_{1}$. 


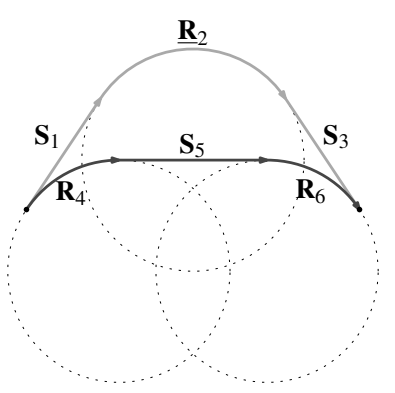

Fig. 3 The $\mathcal{S C} \underline{\mathcal{S}}$ case: $\mathbf{S}_{1} \underline{\mathbf{R}}_{2} \mathbf{S}_{3}$ shown in lighter grey, $\mathbf{R}_{4} \mathbf{S}_{5} \mathbf{R}_{6}$ shown in darker grey

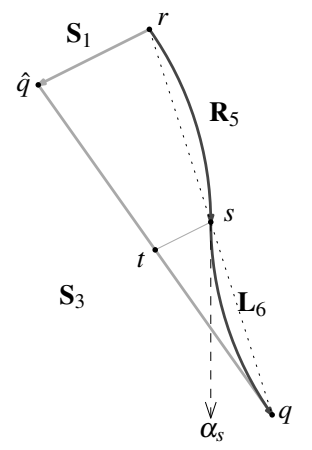

Fig. 5 The $\mathcal{S} \overline{\mathcal{C}} \mathcal{S}$ case: Enlarged diagram with $\mathbf{S}_{1} \mathbf{S}_{3}$ shown in lighter grey, $\mathbf{R}_{5} \mathbf{L}_{6}$ shown in darker grey

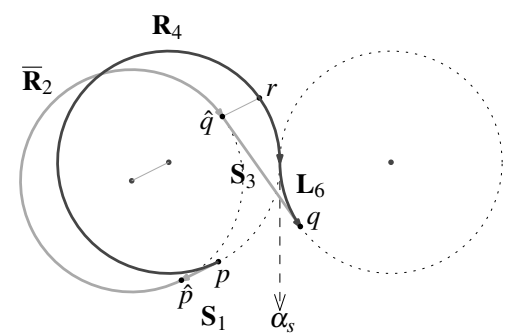

Fig. 4 The $\mathcal{S} \overline{\mathcal{C}} \mathcal{S}$ case: $\mathbf{S}_{1} \overline{\mathbf{R}}_{2} \mathbf{S}_{3}$ shown in lighter grey, $\mathbf{R}_{4} \mathbf{L}_{6}$ shown in darker grey

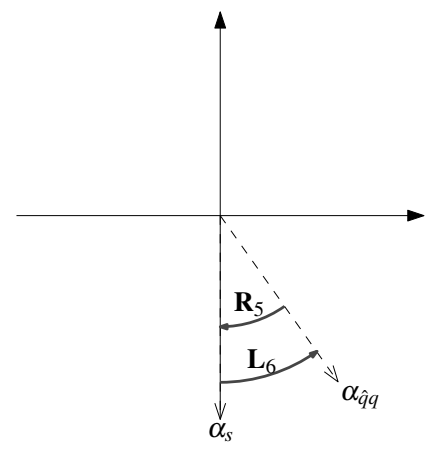

Fig. 6 The $\mathcal{S} \overline{\mathcal{C}} \mathcal{S}$ case: Direction plot of $\mathbf{R}_{5} \mathbf{L}_{6}$ showing $\mathbf{R}_{5}$ and $\mathbf{L}_{6}$ traversing the same directions

\section{Corollary 2 If $v$ is $S P C$, then any $\mathcal{C C C}$ path is not optimal.}

Proof This result follows from similar applying arguments to those in Lemma 4 for the $\mathcal{S C} \mathcal{S}$ case by taking a sufficiently small subpath of the $\mathcal{C} \underline{\mathcal{C}}$ path.

Lemma 5 If $v$ is SPC, then any $\mathcal{C C C C}$ path is not optimal.

Proof From Corollary 2, only $\overline{\mathcal{C C} C}$ cases need to be considered. Without loss of generality, consider $\mathcal{L} \overline{\mathcal{R} L} \mathcal{R}$ paths from $p$ to $q$. We will refer to the polygonal path abcd as a valid encoding if it corresponds to the centres of the circles corresponding to the arcs of a $\mathcal{L} \overline{\mathcal{R} \mathcal{L}} \mathcal{R}$ path from $p$ to $q$ traversed in order. Observe that there is no unique $\mathcal{L} \overline{\mathcal{R} \mathcal{L}} \mathcal{R}$ path from $p$ to $q$, because even though $a$ and $d$ are fixed, the positions of $b$ and $c$ have one degree of freedom. Consider a path $E_{0}=\mathbf{L}_{1} \overline{\mathbf{R}}_{2} \overline{\mathbf{L}}_{3} \mathbf{R}_{4}$ with $b=b_{0}$ and $c=c_{0}$ as shown in Figure 7. By translation, rotation, reflection, and reversing of the path, and corresponding rotation and possibly reflection of $v(\alpha)$, we can assume that $\alpha_{b_{0} c_{0}}=0, a=(0,0), d=(2 h, 2 k)$ and $\alpha_{a b_{0}} \geq \alpha_{c_{0} d}$.

We first consider the case when $\alpha_{a b_{0}} \neq \alpha_{c_{0} d}$ so $\alpha_{a b_{0}}>\alpha_{c_{0} d}$ and look at the effect of a positive increase on $\alpha_{a b}$. It can be seen that any valid encoding abcd must satisfy the conditions in (14)

$$
\begin{array}{r}
\cos \alpha_{a b}+\cos \alpha_{b c}+\cos \alpha_{c d}=h \\
\sin \alpha_{a b}+\sin \alpha_{b c}+\sin \alpha_{c d}=k
\end{array}
$$




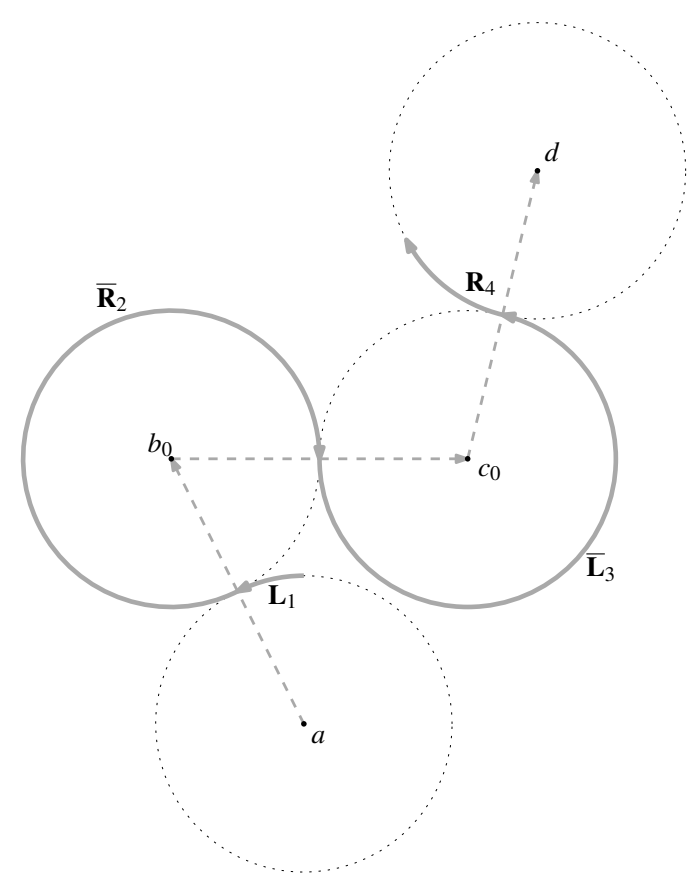

Fig. $7 E_{0}=\mathbf{L}_{1} \overline{\mathbf{R}}_{2} \overline{\mathbf{L}}_{3} \mathbf{R}_{4}$ with corresponding valid encoding $a b_{0} c_{0} d$

Since we are interested in how $\alpha_{b c}$ and $\alpha_{c d}$ change as a result of a positive increase in $\alpha_{a b}$, we implicitly differentiate (14) to obtain (15), where $\mathrm{d} \alpha_{a b}>0$.

$$
\begin{array}{r}
\mathrm{d} \alpha_{a b} \sin \alpha_{a b}+\mathrm{d} \alpha_{b c} \sin \alpha_{b c}+\mathrm{d} \alpha_{c d} \sin \alpha_{c d}=0 \\
\mathrm{~d} \alpha_{a b} \cos \alpha_{a b}+\mathrm{d} \alpha_{b c} \cos \alpha_{b c}+\mathrm{d} \alpha_{c d} \cos \alpha_{c d}=0
\end{array}
$$

When $b=b_{0}, c=c_{0},(15)$ yields (16) and (17).

$$
\begin{aligned}
\mathrm{d} \alpha_{a b} \sin \alpha_{a b_{0}}+\mathrm{d} \alpha_{c d} \sin \alpha_{c_{0} d} & =0 \\
\mathrm{~d} \alpha_{c d} & =-\frac{\sin \alpha_{a b_{0}}}{\sin \alpha_{c_{0} d}} \mathrm{~d} \alpha_{a b} \\
\mathrm{~d} \alpha_{a b} \cos \alpha_{a b_{0}}+\mathrm{d} \alpha_{b c}+\mathrm{d} \alpha_{c d} \cos \alpha_{c_{0} d} & =0 \\
\Rightarrow \mathrm{d} \alpha_{a b} \cos \alpha_{a b_{0}}+\mathrm{d} \alpha_{b c}-\cot \alpha_{c_{0} d} \sin \alpha_{a b_{0}} \mathrm{~d} \alpha_{a b} & =0 \\
\mathrm{~d} \alpha_{b c} & =\sin \alpha_{a b_{0}}\left(\cot \alpha_{c_{0} d}-\cot \alpha_{a b_{0}}\right) \mathrm{d} \alpha_{a b}
\end{aligned}
$$

First, observe from (16) that since $\alpha_{a b_{0}}, \alpha_{c_{0} d} \in(0, \pi)$ and $\mathrm{d} \alpha_{a b}>0$, we have that $\mathrm{d} \alpha_{c d}<$ 0 . Next, since cot is strictly monotonically decreasing on $(0, \pi)$ and $\alpha_{a b_{0}}>\alpha_{c_{0} d}$, it follows from (17) that $\mathrm{d} \alpha_{b c}>0$. Note that the conditions for this argument to hold are that $\alpha_{a b}-$ $\alpha_{b c}, \alpha_{c d}-\alpha_{b c} \in(0, \pi)$ and $\alpha_{a b}-\alpha_{b c}>\alpha_{c d}-\alpha_{b c}$. Also, if it is met for some $b_{1}, c_{1}$, then it is true for some $b_{2}, c_{2}$ where $\alpha_{a b_{2}}=\alpha_{a b_{1}}+\delta$ and $\delta>0$ since $\mathrm{d} \alpha_{a b}>0$ and $\mathrm{d} \alpha_{c d}<0$.

Since $\mathbf{L}_{1} \overline{\mathbf{R}}_{2} \overline{\mathbf{L}}_{3} \mathbf{R}_{4}$ has non-degenerate components, $\exists \gamma_{a b}>0$ such that $a b_{\gamma} c_{\gamma} d$ is a valid encoding, where $\alpha_{a b_{\gamma}}=\alpha_{a b_{0}}+\gamma_{a b}$ as shown in Figure 8. Let the path corresponding to the 


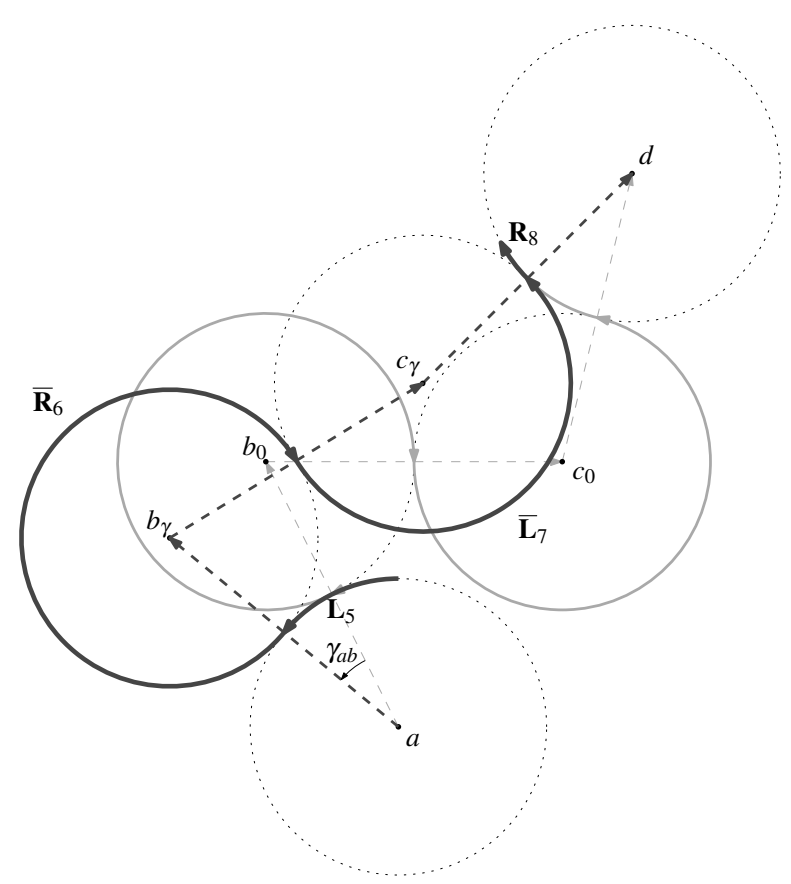

Fig. $8 E_{\gamma}=\mathbf{L}_{5} \overline{\mathbf{R}}_{6} \overline{\mathbf{L}}_{7} \mathbf{R}_{8}$ with corresponding valid encoding $a b_{\gamma} c_{\gamma} d$

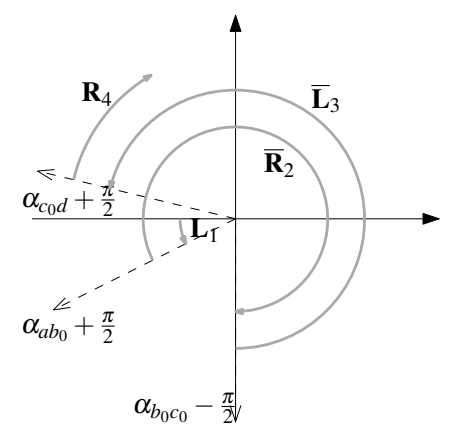

Fig. 9 Direction plot of $E_{0}$

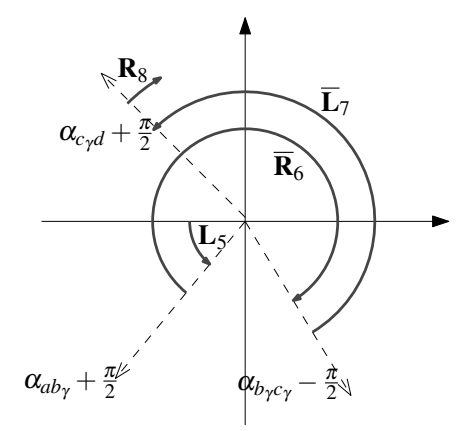

Fig. 10 Direction plot of $E_{\gamma}$

valid encoding $a b_{\gamma} c_{\gamma} d$ be $E_{\gamma}=\mathbf{L}_{5} \overline{\mathbf{R}}_{6} \overline{\mathbf{L}}_{7} \mathbf{R}_{8}$. Since the conditions are met when $b=b_{0}$ and $c=c_{0}$, it then follows that $\gamma_{a b}, \gamma_{b c}>0$ and $\gamma_{c d}<0$ where $\gamma_{b c}=\alpha_{b_{\gamma} c_{\gamma}}-\alpha_{b_{0} c_{0}}$ and $\gamma_{c d}=$ $\alpha_{c \gamma d_{\gamma}}-\alpha_{c_{0} d_{0}}$.

Now we need to show that $T\left(E_{\gamma}\right)<T\left(E_{0}\right)$. Let $\mathbf{L}_{i, j}$ represent $\mathbf{L}_{i} \backslash \mathbf{L}_{j}$ and $\mathbf{R}_{i, j}$ represent $\mathbf{R}_{i} \backslash \mathbf{R}_{j}$. Note that $\mathbf{L}_{3,7}$ contains two disjoint $\mathcal{L}$ arcs as shown in Figure 12. By comparing the direction plots shown in Figures 9, 10 and 11, it can be seen that what needs to be shown is $T\left(\mathbf{L}_{5,1}\right)+T\left(\mathbf{R}_{6,2}\right)<T\left(\mathbf{R}_{2,6}\right)+T\left(\mathbf{L}_{3,7}\right)+T\left(\mathbf{R}_{4,8}\right)$ as shown in Figure 12. It is also clear from the direction plot that $T\left(\mathbf{R}_{6,2}\right)=T\left(\mathbf{L}_{5,1}\right)$ and $T\left(\mathbf{R}_{2,6}\right)+T\left(\mathbf{R}_{4,8}\right)=T\left(\mathbf{L}_{3,7}\right)$. Since translating paths do not change their costs, it can be seen from Figure 12 that Lemma 3 can now be applied to obtain $T\left(\mathbf{L}_{5,1}\right)<T\left(\mathbf{L}_{3,7}\right)$, which gives $T\left(E_{\gamma}\right)<T\left(E_{0}\right)$. 


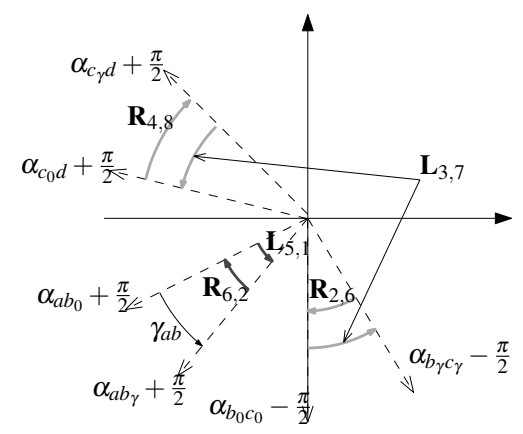

Fig. 11 Direction plot of the difference between $E_{0}$ and $E_{\gamma}$

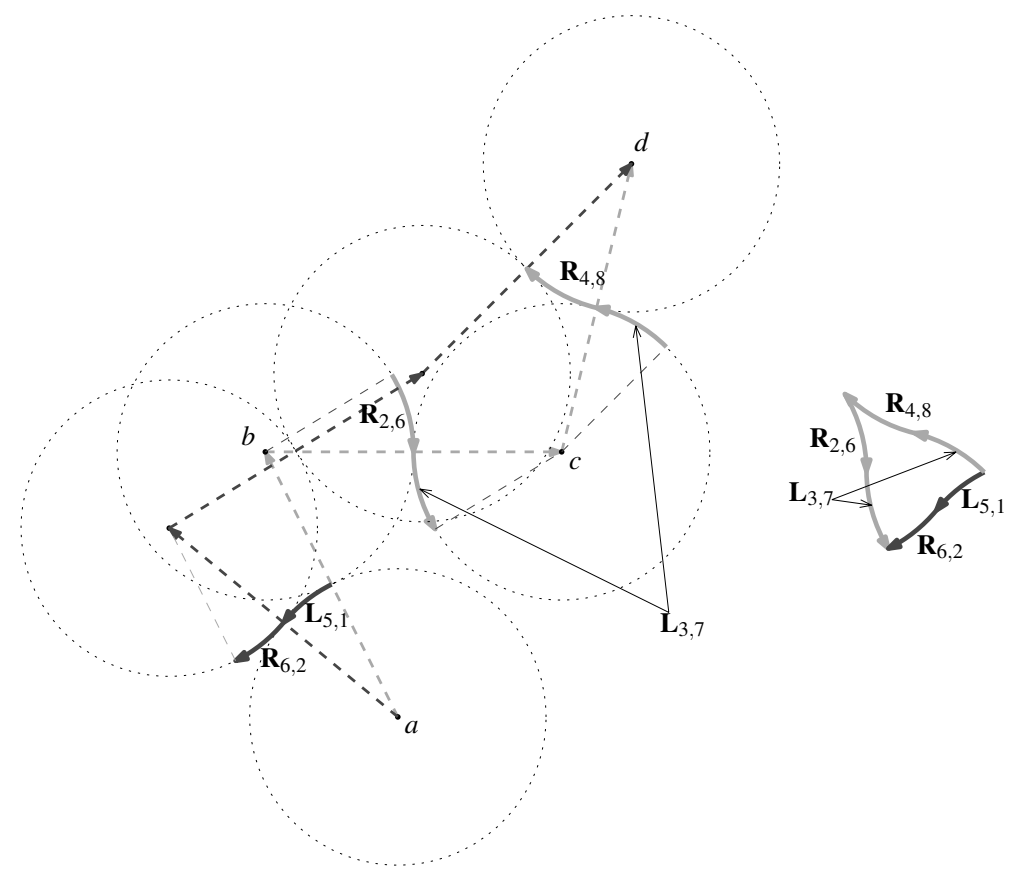

Fig. 12 Difference between $E_{0}$ and $E_{\gamma}$

Angle $\alpha_{a b}$ can continue to be increased until either $\mathbf{R}_{4}$ becomes degenerate or $\mathbf{R}_{2}$ or $\mathbf{L}_{3}$ traverses an angle of $\pi$.

Clearly, it follows that deforming the path in the opposite direction which deforms the path towards achieving $\alpha_{a b}=\alpha_{c d}$ never decreases the cost. Hence, the configuration where $\alpha_{a b}=\alpha_{c d}$ is a local maximum among $\mathcal{C C C C}$ paths with fixed endpoints, by symmetry.

In the case of $(v(\alpha)=1)$, this result was proven by Dubins [5] and Boissonnat et al. [1] using different techniques. However, both proofs were not suitable for extending to the SPC $v$ case because there was no easy way to make use of the strict triangle inequality. The proof above makes use of the strict triangle inequality to extend the result to a general SPC $v$ case.

Finally, putting all of the results together gives the following theorem. 
Theorem 3 If $v$ is SPC, then any optimal path is a Dubins path.

We can now apply Theorem 3, to obtain the following result for weakly polarly convex (WPC) functions.

Corollary 3 If $v$ is WPC, then an optimal curvature-constrained directional-cost path can be found by considering only Dubins paths.

Proof Consider a WPC velocity function $v$, where $c=1 / v$. Define a new velocity function $v_{\varepsilon}=1 / c_{\varepsilon}$ by $c_{\varepsilon}=c+\varepsilon$. Using Property 1 , we are able to show that $v_{\varepsilon}$ is SPC for any $\varepsilon>0$ by using the fact that $v$ is WPC as follows. Clearly $c$ and $c_{\varepsilon}$ are twice differentiable on the same intervals of $\alpha$, and where they are twice differentiable,

$$
c_{\varepsilon}^{\prime \prime}+c_{\varepsilon}=c^{\prime \prime}+c+\varepsilon \geq \varepsilon>0
$$

The second condition obviously holds since $c^{\prime}(\alpha)=c_{\varepsilon}^{\prime}(\alpha)$. This shows that $v_{n}$ is PC and strict, and hence is SPC. Let $T(E)$ and $T_{\mathcal{E}}(E)$ denote the costs of path $E$ with respect to the directional-cost functions $c$ and $c_{\varepsilon}$ respectively. Let $p, q$ be any arbitrary start and end directed points. Let $\mathbb{D}_{p q}$ denote the set of all Dubins paths from $p$ to $q$ as described in Section 2, and recall that $\mathbb{P}_{p q}$ is the set of all curvature-constrained paths from $p$ to $q$.

Suppose $\exists E \in \mathbb{P}_{p q} \backslash \mathbb{D}_{p q}$ such that $T(E)<T(D) \forall D \in \mathbb{D}_{p q}$. Then,

$$
\begin{aligned}
T_{\varepsilon}(E) & =T(E)+\varepsilon L(E) \\
& <T(D), \text { by choosing } \varepsilon \in(0,(T(D)-T(E)) /(L(E))) \\
& <T_{\mathcal{E}}(D), \forall D \in \mathbb{D}_{p q}
\end{aligned}
$$

However, Theorem 3 states that there cannot exist such a $E$ since $v_{\varepsilon}$ is SPC and $E \notin \mathbb{D}_{p q}$. Hence, by contradiction, we know that there cannot exist a non-Dubins path which is of less cost than all other Dubins paths, for any WPC velocity function.

From Theorem 3, it follows that given directed points $p, q$, the minimum length curvatureconstrained path and the optimal curvature-constrained directional-cost path are both Dubins paths if $v$ is SPC. However, Example 1 illustrates that they do not have to be the same path.

Example 1 Let $v(\alpha)$ be defined as in (18) (shown in Figure 13).

$$
v(\alpha)=\left\{\begin{aligned}
\frac{4}{9 \pi} \alpha^{3}+1, & \alpha \in\left[0, \frac{3 \pi}{8}\right] \\
\frac{4}{9 \pi}\left(\frac{3 \pi}{4}-\alpha\right)^{3}+1, & \alpha \in\left(\frac{3 \pi}{8}, \frac{3 \pi}{4}\right) \\
1, & \alpha \in\left(\frac{3 \pi}{4}, 2 \pi\right)
\end{aligned}\right.
$$

By Property 1 , it is easily checked that $v(\alpha)$ is SPC. Let $p=(0,0, \pi)$ and $q=(-2 \sqrt{2}, 0,0)$ be the start and end directed points respectively. The resulting paths $\mathbf{L}_{1} \mathbf{S}_{2} \mathbf{R}_{3}$ and $\mathbf{R}_{4} \mathbf{S}_{5} \mathbf{L}_{6}$ are of equal length as shown in Figure 14. However, the direction plots shown in Figures 15 and 16 illustrate that they traverse different directions. Hence, $T\left(\mathbf{L}_{1} \mathbf{S}_{2} \mathbf{R}_{3}\right)<L\left(\mathbf{L}_{1} \mathbf{S}_{2} \mathbf{R}_{3}\right)=$ $L\left(\mathbf{R}_{4} \mathbf{S}_{5} \mathbf{L}_{6}\right)=T\left(\mathbf{R}_{4} \mathbf{S}_{5} \mathbf{L}_{6}\right)$. Clearly, there exists $\varepsilon>0$ such that the shortest curvature-constrained path from $\hat{p}=(0, \varepsilon, \pi)$ to $q$ is an $\mathcal{R S} \mathcal{L}$ path while the optimal path from $\hat{p}$ to $q$ is an $\mathcal{L} \mathcal{S}$ path. 


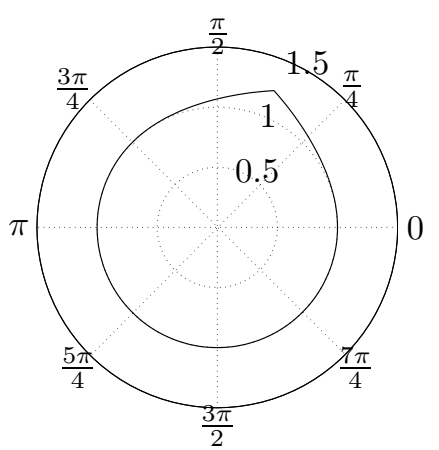

Fig. $13 v(\alpha)$ as specified in (18)

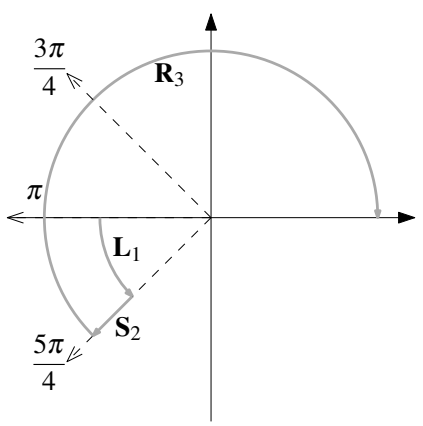

Fig. 15 Direction plot of $\mathbf{L}_{1} \mathbf{S}_{2} \mathbf{R}_{3}$

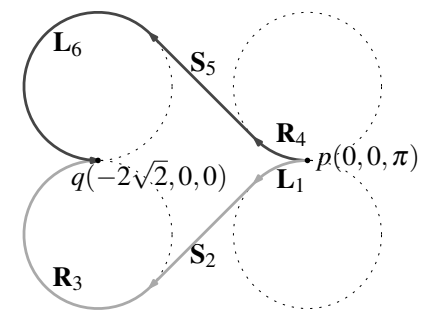

Fig. $14 \mathbf{L}_{1} \mathbf{S}_{2} \mathbf{R}_{3}$ and $\mathbf{R}_{4} \mathbf{S}_{5} \mathbf{L}_{6}$

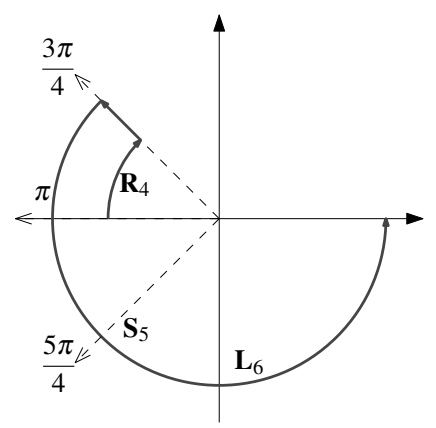

Fig. 16 Direction plot of $\mathbf{R}_{4} \mathbf{S}_{5} \mathbf{L}_{6}$

\section{Conclusion}

The mathematical problem studied was motivated by the effects of directional faulting on development cost of underground mine declines. In particular, this meant extending Dubins [5] result of minimal length paths, to incorporate a directional-cost element. It was shown that if the velocity function is strictly polarly convex, any optimal path is a Dubins paths. If the velocity function is polarly convex, then there exists an optimal path which is a Dubins path. The results proved in this paper lay the foundation for future works developing the theory necessary for the design of underground mines taking into consideration anisotropic development and support costs. It has also been seen that it is a useful problem to consider for other practical applications such as naval path planning [4].

From a theoretical viewpoint, this result provides a more general context for the Dubins result, in that Dubins paths are actually optimal paths for a much more general problem where the velocity depends on the direction, provided that the velocity function is polarly convex.

This paper is the first in a series of papers which will develop the theory necessary to implement an efficient algorithm for constructing optimal underground mine network designs in anisotropic ground conditions. The most immediate question which was not yet addressed in this paper are what the forms of optimal paths are when subject to a polarly non-convex velocity function. The results of this next step will be presented in a future paper. Another extension will be to consider inhomogeneity through geological domains 
with different directional-cost functions which is related to the problem studied in [11]. In order to construct feasible paths for underground mine networks, the problem of lifting these planar paths into 3-d while satisfying a gradient constraint will need be studied such as in [2].

Acknowledgements This research is supported by a grant from the Australian Research Council.

\section{References}

1. J.D. Boissonnat, A. Cerezo, and J. Leblond, Shortest paths of bounded curvature in the plane, J. Intell. Robot. Syst. 11 (1994), 5-20.

2. M. Brazil, P.A. Grossman, D.H. Lee, J.H. Rubinstein, D.A. Thomas, and N.C. Wormald, Decline design in underground mines using constrained path optimisation, Min. Technol.: Trans. Inst. Min. Metall., Sect. A 117 (2008), no. 2, 93-99.

3. X.N. Bui, P. Soueres, J.D. Boissonnat, and J.P. Laumond, The shortest path synthesis for non-holonomic robots moving forwards, INRIA, Nice-Sophia-Antipolis, Research Report 2153, 1993.

4. I.S. Dolinskaya, Optimal path finding in direction, location and time dependent environments, Ph.D. thesis, Industrial and Operations Engineering, The University of Michigan, 2009.

5. L.E. Dubins, On curves of minimal length with a constraint on average curvature, and with prescribed initial and terminal positions and tangents, Am. J. Math. 79 (1957), 497-516.

6. K. Gehring and M. Fuchs, Quantification of rock mass influence on cuttability with roadheaders, 28th ITA (International Tunnelling Association) General Assembly and World Tunnel Congress. Sydney, 2002.

7. D.H. Laubscher, A geomechanics classification system for the rating of rock mass in mine design, J. S. Atr. Inst. Min. Metal 90 (1990), no. 10, 257-273.

8. T.G. McGee, S.Spry, and J.K. Hedrick, Optimal path planning in a constant wind with a bounded turning rate, Proceedings of the AIAA Conference on Guidance, Navigation and Control, Ketstone, Colorado (2005).

9. L.S. Pontryagin, The mathematical theory of optimal processes, vol. 4, Interscience, 1962, Translation of a Russian book.

10. J. A. Reeds and L. A. Shepp, Optimal paths for a car that goes both forwards and backwards, Pacific Journal of Mathematics 145 (1990), no. 2, 367-393.

11. R.G. Sanfelice and E. Frazzoli, On the optimality of dubins paths across heterogeneous terrain, Hybrid Systems: Computation and Control, vol. 4981, Springer Berlin / Heidelberg, 2008, pp. 457-470.

12. A.M. Shkel and V. Lumelsky, Classification of the dubins set, Robotics and Autonomous Systems 34 (2001), no. 4, $179-202$.

13. P. Soueres and J.P. Laumond, Shortest paths synthesis for a car-like robot, IEEE Transactions on Automatic Control 41 (1996), no. 5, 672-688. 


\section{University Library}

\section{- M M I N E R VA A gateway to Melbourne's research publications}

Minerva Access is the Institutional Repository of The University of Melbourne

Author/s:

Chang, AJ;Brazil, M;Rubinstein, JH;Thomas, DA

Title:

Curvature-constrained directional-cost paths in the plane

Date:

2012-08-01

Citation:

Chang, A. J., Brazil, M., Rubinstein, J. H. \& Thomas, D. A. (2012). Curvature-constrained directional-cost paths in the plane. JOURNAL OF GLOBAL OPTIMIZATION, 53 (4), pp.663-681. https://doi.org/10.1007/s10898-011-9730-1.

Persistent Link:

http://hdl.handle.net/11343/283174 